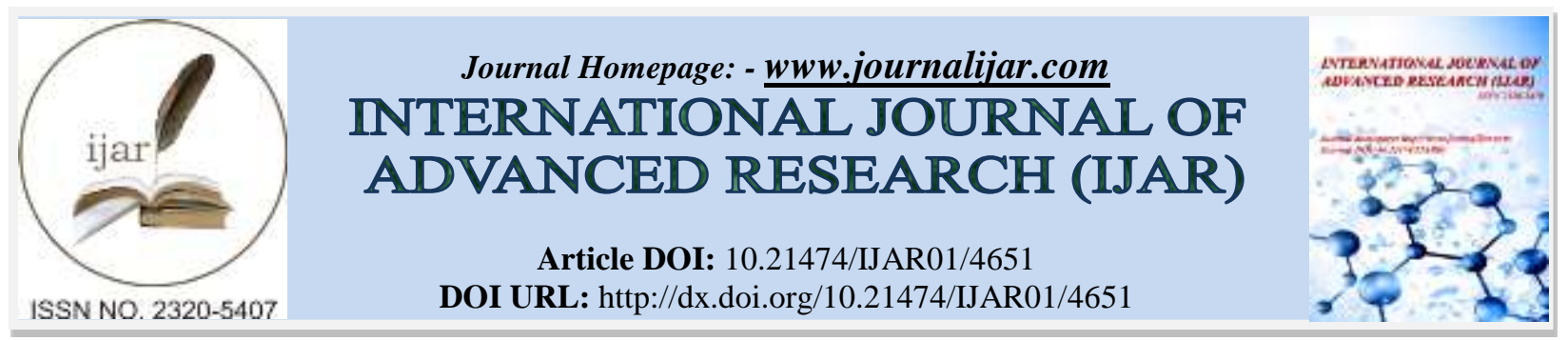

RESEARCH ARTICLE

\title{
SYNTHESIS AND CHARACTERIZATION OF SCHIFF BASES AND THIAZOLIDINONE DERIVATIVES AND THEIR MICROBIAL EVALUATION.
}

\author{
Savan V. Patel ${ }^{1}$, Rahul B. Tailor ${ }^{2}$, Sejal M. Patel ${ }^{2}$, K. C. Patel ${ }^{2}$ and K. D. Prajapati ${ }^{1}$. \\ 1. V. S. Patel College of Arts and Science Bilimora. \\ 2. Department of Chemistry, Veer Narmad South Gujarat University Surat, Gujarat, India.
}

\section{Manuscript Info}

(.........................

Manuscript History

Received: 28 April 2017

Final Accepted: 30 May 2017

Published: June 2017

Key words:-

Schiff bases, thiazolidinone,

antibacterial, thioglycolic acid, antifungal

\section{Abstract}

A series of 4,4'-Methylene bis (N-substituted benzylidene-2-methyl5-nitro aniline) derivatives ( Schiff bases ) $\mathrm{Sa}_{1}$ to $\mathrm{Sa}_{10}$ were prepared by the condensation of 4 4'- methylene bis(2-methyl-5-nitro aniline) with different aromatic aldehyde. Treatment of $\mathrm{Sa}_{1}$ to $\mathrm{Sa}_{10}$ with thioglycolic acid led to the formation of thiazolidinone derivatives $\mathrm{Sb}_{1}$ to $\mathrm{Sb}_{10}$. All the synthesized compounds characterized by FTIR, ${ }^{1} \mathrm{H}$ NMR and Elemental analysis. The antibacterial activity of these compounds was first studied in vitro by the disk diffusion assay against gram positive and gram negative bacteria. The minimum inhibition concentration (MIC) was then determined with the references of standard drug ampicillin. The antifungal activity was examined against Candida albicans and Aspergillus niger by the serial plate dilution method using fluconazole as standard.

Copy Right, IJAR, 2017,. All rights reserved.

\section{Introduction:-}

Schiff bases are the compounds containing azomethine group $(-\mathrm{HC}=\mathrm{N}-)$ which were first reported by Hugo Schiff in 1864 and formed by condensation of a primary amine with an active carbonyl compound, and generally take place under acid, base catalysis or with heat[1]. Schiff bases are useful chelators because of their ease of preparation, structural varieties, varied denticities and subtle steric and electronic control on their framework [2]. Schiff bases have potential sites, such as nitrogen and other donors; it may be attributed to their stability and applications in many fields [3]. They are considered as "privileged ligands" and are most widely used due to their versatile synthesis and good solubility in common solvents [4]. In azomethine derivatives, the $\mathrm{C}=\mathrm{N}$ linkage is essential for biological activity. The nitrogen atom of azomethine may be involved in the formation of constituents and interferes in normal cell processes Schiff-bases are every important material for inorganic chemists as these are widely used in medicinal inorganic chemistry due to their diverse biological, pharmacological, antitumor activities. Schiff-bases have gained much importance in biomimetic modelling applications, designing molecular magnet molecules, and in liquid crystals aspect [5]. Heterocyclic scaffolds containing an azole ring system and phenol derivatives have been known to possess a wide range of biological application such as antifungal[6], antioxidant[7], antibacterial[8], antitumor[9], anti-inflammatory[10] and antipyretic applications[11].

4-thiazolidinone has been considered as a magic moiety (wonder nucleus) and represents an important class of heterocyclic compounds with a wide spread biological applications. Thiazolidinone derivatives have been investigated for a range of pharmacologic indications such as anti-viral[12], anti-convulsant[13], 
cardiovascular[14], anti-inflammatory[15], antidiabetic[16], antihyperlipidemic[17], antimicrobial[18], antituberculosis[19], antiparasitis[20], FSH agonist [21], antiarthritic [22 ] and antidiarrhoeal[23] activity.

\section{Material and Methods:-}

\section{Instrumentation and chemicals:-}

All the chemicals and solvent used for this work obtained from sigma Aldrich and loba chem. Melting point of the synthesized compounds were determined in open glass capillaries on stuart SMP10 appartus. IR absorption spectra were recorded on shimadzu FTIR 8400s using $\mathrm{KBr}$ pellets in the range of $4000-400 \mathrm{~cm}^{-1}$. ${ }^{1} \mathrm{H}$ NMR spectra were recorded on the bruker spectrometer operating at $300 \mathrm{MHz}$ and TMS. Purity of the compound are checked by thin layer chromatography on merck silica gel $60 \mathrm{~F}_{254}$ precoated sheets in methanol mixture and spot were developed using iodine chamber.

\section{Biological Activity:-}

All the newly synthesized compounds $\mathrm{Sa}_{1}-\mathrm{Sb}_{10}$ were evaluated for their in vitro antibacterial and antifungal activities. The antibacterial activity was carried out against two Gram-negative bacteria (Escherichia coli ATCC 25922 and Pseudomonas aeruginosa ATCC 27853) and two Gram-positive bacteria (Staphylococcus aureus ATCC 25923 and Bacillus subtilis ATCC 6633) by the test tube dilution technique using Mueller-Hinton nutrient broth (Jones et al., 1991). Ciprofloxacin and ampicillin were used as a reference standard. The antifungal activity was examined against Candida albicans (NCIM No. 3471) and Aspergillus niger (NCIM 1196) by the serial plate dilution method (Khan, 1997) using fluconazole as standard.

\section{Experimental protocol:-}

Synthesis:-

General procedure for the synthesis of Schiff bases:-

A mixture of two mole of substituted aldehyde and one mole of 4-4'-methylene bis(2-methyl-5-nitro aniline) in methanol was refluxed for 8-10 $\mathrm{h}$ in the presence of few drops of glacial acetic acid as catalyst. The progress of reaction was monitored by $\mathrm{TlC}$ at appropriate time interval. After completion of reaction the solution was cooled, solid thus separated was washed with ice-cold water and dried, finally, the product thus obtained was recrystallized from ethanol.

3.1.1.1. 4-4'-methylene bis(2-chloro benzylidene-2-methyl-5-nitro aniline) $\left(\mathrm{Sa}_{1}\right)$. Light yellow crystals; yield: $72 \%$; $\mathrm{mp} 172-175^{\circ} \mathrm{C} ; R_{f} 0.64$ (ethyl acetate:ammonia, 9:0.01); IR $\left(\mathrm{KBr}, \mathrm{cm}^{-1}\right): 3324(\mathrm{NH}), 1544(\mathrm{C}=\mathrm{N}), 1077(\mathrm{C}-\mathrm{N}), 2890$ $(\mathrm{C}-\mathrm{H}), 1510(\mathrm{~N}=\mathrm{O}), 680(\mathrm{C}-\mathrm{Cl}) ;{ }^{1} \mathrm{H}$ NMR (DMSO, ppm): $\delta 4.45$ (s, 2H, $\mathrm{CH}_{2}$ ), 8.56 (s, 2H, Schiff Base), 7.30-7.82 (m, $12 \mathrm{H}, \mathrm{Ar}-\mathrm{H}), 2.34$ (s, $6 \mathrm{H}, \mathrm{CH}_{3}$ ); EI-MS: $\mathrm{m} / z[\mathrm{M}+\mathrm{H}]^{+}$561; Anal. Calcd for $\mathrm{C}_{29} \mathrm{H}_{22} \mathrm{Cl}_{2} \mathrm{~N}_{4} \mathrm{O}_{4}: \mathrm{C}, 62.04 ; \mathrm{H}, 3.95 ; \mathrm{N}$, 9.98. Found: C, 62.09; H, 3.88; N, 9.93.

3.1.1.2. 4-4'-methylene bis(4-chloro benzylidene-2-methyl-5-nitro aniline) $\left(\mathrm{Sa}_{2}\right)$. Light yellow crystals; yield: 78\%; $\mathrm{mp} 168-170^{\circ} \mathrm{C} ; R_{f} 0.70$ (ethyl acetate:ammonia, 9:0.01); IR $\left(\mathrm{KBr}, \mathrm{cm}^{-1}\right): 3360(\mathrm{NH}), 1540(\mathrm{C}=\mathrm{N}), 1082(\mathrm{C}-\mathrm{N}), 2960$ $(\mathrm{C}-\mathrm{H}), 1520(\mathrm{~N}=\mathrm{O}), 670(\mathrm{C}-\mathrm{Cl}) ;{ }^{1} \mathrm{H}$ NMR (DMSO, ppm): $\delta 4.62\left(\mathrm{~s}, 2 \mathrm{H}, \mathrm{CH}_{2}\right), 8.46$ (s, 2H, Schiff Base), 7.27-7.76 (m, $12 \mathrm{H}, \mathrm{Ar}-\mathrm{H}), 2.34$ (s, $6 \mathrm{H}, \mathrm{CH}_{3}$ ); EI-MS: $\mathrm{m} / z[\mathrm{M}+\mathrm{H}]^{+}$562; Anal. Calcd for $\mathrm{C}_{29} \mathrm{H}_{22} \mathrm{Cl}_{2} \mathrm{~N}_{4} \mathrm{O}_{4}: \mathrm{C}, 62.04 ; \mathrm{H}, 3.95 ; \mathrm{N}$, 9.98. Found: C, 62.09; H, 3.88; N, 9.93.

3.1.1.3. 4-4'-methylene bis(4-methyl benzylidene-2-methyl-5-nitro aniline) ( $\mathrm{Sa}_{3}$ ). Light yellow crystals; yield:

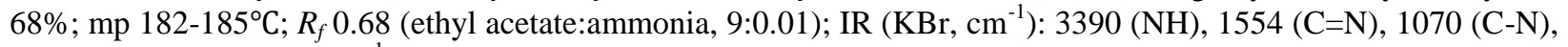
$2990(\mathrm{C}-\mathrm{H}), 1490(\mathrm{~N}=\mathrm{O}) ;{ }^{1} \mathrm{H}$ NMR (DMSO, ppm): $\delta 4.62\left(\mathrm{~s}, 2 \mathrm{H}, \mathrm{CH}_{2}\right), 8.36$ (s, 2H, Schiff Base), 7.37-7.96 (m, $12 \mathrm{H}, \mathrm{Ar}-\mathrm{H}), 2.34-2.52\left(\mathrm{~m}, 12 \mathrm{H}, \mathrm{CH}_{3}\right)$; EI-MS: $\mathrm{m} / z[\mathrm{M}+\mathrm{H}]^{+} 492$; Anal. Calcd for $\mathrm{C}_{29} \mathrm{H}_{24} \mathrm{~N}_{4} \mathrm{O}_{4}: \mathrm{C}, 70.72 ; \mathrm{H}, 4.91 ; \mathrm{N}$, 11.38. Found: C, 70.79; H, 4.84; N, 11.27

3.1.1.4. 4-4'-methylene bis(2-fluoro benzylidene-2-methyl-5-nitro aniline) ( $\left.\mathrm{Sa}_{4}\right)$. Yellow crystals; yield: $84 \%$; $\mathrm{mp}$ $192-193^{\circ} \mathrm{C} ; R_{f} 0.75$ (ethyl acetate:ammonia, 9:0.01); IR $\left(\mathrm{KBr}, \mathrm{cm}^{-1}\right): 3370(\mathrm{NH}), 1550(\mathrm{C}=\mathrm{N}), 1080(\mathrm{C}-\mathrm{N}), 2970(\mathrm{C}-$ $\mathrm{H}), 1470(\mathrm{~N}=\mathrm{O}), 720(\mathrm{C}-\mathrm{F}) ;{ }^{1} \mathrm{H}$ NMR (DMSO, ppm): $\delta 4.62\left(\mathrm{~s}, 2 \mathrm{H}, \mathrm{CH}_{2}\right), 8.72$ (s, 2H, Schiff Base), 7.17-7.86 (m, $12 \mathrm{H}, \mathrm{Ar}-\mathrm{H}), 2.34\left(\mathrm{~m}, 6 \mathrm{H}, \mathrm{CH}_{3}\right)$; EI-MS: $\mathrm{m} / z[\mathrm{M}+\mathrm{H}]^{+}$528; Anal. Calcd for $\mathrm{C}_{29} \mathrm{H}_{22} \mathrm{~F}_{2} \mathrm{~N}_{4} \mathrm{O}_{4}: \mathrm{C}, 65.91 ; \mathrm{H}, 4.20$; $\mathrm{N}$, 10.60. Found: C, $65.85 ; \mathrm{H}, 4.28 ; \mathrm{N}, 10.68$. 
3.1.1.5. 4-4'-methylene bis(4-fluoro benzylidene-2-methyl-5-nitro aniline) ( $\left.\mathrm{Sa}_{5}\right)$. Yellow crystals; yield: 76\%; $\mathrm{mp}$ 210-212 ${ }^{\circ} \mathrm{C} ; R_{f} 0.66$ (ethyl acetate:ammonia, 9:0.01); IR $\left(\mathrm{KBr}, \mathrm{cm}^{-1}\right): 3365(\mathrm{NH}), 1545(\mathrm{C}=\mathrm{N}), 1075(\mathrm{C}-\mathrm{N}), 2960(\mathrm{C}-$ $\mathrm{H}), 1520(\mathrm{~N}=\mathrm{O}), 710(\mathrm{C}-\mathrm{F}) ;{ }^{1} \mathrm{H}$ NMR (DMSO, ppm): $\delta 4.62\left(\mathrm{~s}, 2 \mathrm{H}, \mathrm{CH}_{2}\right), 8.62(\mathrm{~s}, 2 \mathrm{H}$, Schiff Base), 7.40-7.92 (m, $12 \mathrm{H}, \mathrm{Ar}-\mathrm{H}), 2.34\left(\mathrm{~m}, 6 \mathrm{H}, \mathrm{CH}_{3}\right)$; EI-MS: $\mathrm{m} / z[\mathrm{M}+\mathrm{H}]^{+}$528; Anal. Calcd for $\mathrm{C}_{29} \mathrm{H}_{22} \mathrm{~F}_{2} \mathrm{~N}_{4} \mathrm{O}_{4}: \mathrm{C}, 65.91 ; \mathrm{H}, 4.20 ; \mathrm{N}$, 10.60. Found: C, $65.85 ; \mathrm{H}, 4.28 ; \mathrm{N}, 10.68$.

3.1.1.6. 4-4'-methylene bis(2-nitro benzylidene-2-methyl-5-nitro aniline) ( $\left.\mathbf{S} \boldsymbol{a}_{6}\right)$. Yellow crystals; yield: 83\%; $\mathrm{mp}$ $189-190^{\circ} \mathrm{C} ; R_{f} 0.78$ (ethyl acetate:ammonia, 9:0.01); IR $\left(\mathrm{KBr}, \mathrm{cm}^{-1}\right): 3380(\mathrm{NH}), 1535(\mathrm{C}=\mathrm{N}), 1065(\mathrm{C}-\mathrm{N}), 2980(\mathrm{C}-$ $\mathrm{H}), 1535(\mathrm{~N}=\mathrm{O}) ;{ }^{1} \mathrm{H}$ NMR (DMSO, ppm): $\delta 4.62\left(\mathrm{~s}, 2 \mathrm{H}, \mathrm{CH}_{2}\right), 8.60-8.76(\mathrm{~s}, 2 \mathrm{H}$, Schiff Base), 7.30-7.78 (m, 12H, $\mathrm{Ar}-\mathrm{H}), 2.34\left(\mathrm{~m}, 6 \mathrm{H}, \mathrm{CH}_{3}\right)$; EI-MS: $\mathrm{m} / 2[\mathrm{M}+\mathrm{H}]^{+}$582; Anal. Calcd for $\mathrm{C}_{29} \mathrm{H}_{22} \mathrm{~N}_{6} \mathrm{O}_{8}: \mathrm{C}, 59.79 ; \mathrm{H}, 3.81 ; \mathrm{N}, 14.43$. Found: C, 59.64; H, 3.76; N, 14.38 .

3.1.1.7. 4-4'-methylene bis(4-nitro benzylidene-2-methyl-5-nitro aniline) ( $\left.\mathrm{Sa}_{7}\right)$. Yellow crystals; yield: 78\%; mp 220-222 ${ }^{\circ}$; $R_{f} 0.74$ (ethyl acetate:ammonia, 9:0.01); IR $\left(\mathrm{KBr}, \mathrm{cm}^{-1}\right): 3375(\mathrm{NH}), 1550(\mathrm{C}=\mathrm{N}), 1070(\mathrm{C}-\mathrm{N}), 2990(\mathrm{C}-$ $\mathrm{H}), 1530(\mathrm{~N}=\mathrm{O}) ;{ }^{1} \mathrm{H}$ NMR (DMSO, ppm): $\delta 4.62\left(\mathrm{~s}, 2 \mathrm{H}, \mathrm{CH}_{2}\right), 8.42-8.58(\mathrm{~s}, 2 \mathrm{H}$, Schiff Base), 7.42-8.02 (m, 12H, $\mathrm{Ar}-\mathrm{H}), 2.34\left(\mathrm{~s}, 6 \mathrm{H}, \mathrm{CH}_{3}\right)$; EI-MS: $\mathrm{m} / 2[\mathrm{M}+\mathrm{H}]^{+}$582; Anal. Calcd for $\mathrm{C}_{29} \mathrm{H}_{22} \mathrm{~N}_{6} \mathrm{O}_{8}: \mathrm{C}, 59.79 ; \mathrm{H}, 3.81 ; \mathrm{N}, 14.43$. Found: C, 59.64; H, 3.76; N, 14.38 .

3.1.1.8. 4-4'-methylene bis(2-hydroxy benzylidene-2-methyl-5-nitro aniline) ( $\mathrm{Sa} \mathrm{a}_{8}$ ). Light yellow crystals; yield: 70\%; mp $236^{\circ} \mathrm{C} ; R_{f} 0.62$ (ethyl acetate:ammonia, 9:0.01); IR $\left(\mathrm{KBr}, \mathrm{cm}^{-1}\right): 3310(\mathrm{NH}), 1530(\mathrm{C}=\mathrm{N}), 1060(\mathrm{C}-\mathrm{N})$, $2970(\mathrm{C}-\mathrm{H}), 1510(\mathrm{~N}=\mathrm{O}), 3420(\mathrm{O}-\mathrm{H}) ;{ }^{1} \mathrm{H}$ NMR (DMSO, ppm): $\delta 4.62\left(\mathrm{~s}, 2 \mathrm{H}, \mathrm{CH}_{2}\right), 8.92(\mathrm{~s}, 2 \mathrm{H}$, Schiff bases), 6.80-7.72 (m, 12H, Ar-H), $2.34\left(\mathrm{~s}, 6 \mathrm{H}, \mathrm{CH}_{3}\right), 11.11(\mathrm{~s}, 2 \mathrm{H}, \mathrm{OH})$; EI-MS: $\mathrm{m} / z$ [M+H] ${ }^{+}$524; Anal. Calcd for $\mathrm{C}_{29} \mathrm{H}_{24} \mathrm{~N}_{4} \mathrm{O}_{4}$ : C, 66.41; H, 4.61; N, 10.68. Found: C, 66.37; H, 4.68; N, 10.59.

3.1.1.9. 4-4'-methylene bis(2-hydroxy-3-methoxy benzylidene-2-methyl-5-nitro aniline) (Sa $a_{9}$. Light yellow crystals; yield: $72 \%$; mp $203-205^{\circ} \mathrm{C} ; R_{f} 0.70$ (ethyl acetate:ammonia, 9:0.01); IR $\left(\mathrm{KBr}, \mathrm{cm}^{-1}\right.$ ): $3365(\mathrm{NH}), 1540$ $(\mathrm{C}=\mathrm{N}), 1050(\mathrm{C}-\mathrm{N}), 2980(\mathrm{C}-\mathrm{H}), 1520(\mathrm{~N}=\mathrm{O}), 3440(\mathrm{O}-\mathrm{H}) ;{ }^{1} \mathrm{H}$ NMR (DMSO, ppm): $\delta 4.62\left(\mathrm{~s}, 2 \mathrm{H}, \mathrm{CH}_{2}\right), 8.88$ (s, $2 \mathrm{H}$, Schiff bases), $3.80\left(\mathrm{~s}, 6 \mathrm{H}, \mathrm{OCH}_{3}\right), 6.88-7.76(\mathrm{~m}, 10 \mathrm{H}, \mathrm{Ar}-\mathrm{H}), 2.34\left(\mathrm{~s}, 6 \mathrm{H}, \mathrm{CH}_{3}\right), 13.72(\mathrm{~s}, 2 \mathrm{H}, \mathrm{OH})$; EI-MS: $\mathrm{m} / z$ $[\mathrm{M}+\mathrm{H}]^{+}$584; Anal. Calcd for $\mathrm{C}_{31} \mathrm{H}_{28} \mathrm{~N}_{4} \mathrm{O}_{8}: \mathrm{C}, 63.69 ; \mathrm{H}, 4.83 ; \mathrm{N}, 9.58$. Found: C, 63.62; H, 4.72; N, 9.72.

3.1.1.10. 4-4'-methylene bis(4-hydroxy-3-methoxy benzylidene-2-methyl-5-nitro aniline) ( $\left.\mathbf{S} a_{10}\right)$. Light yellow crystals; yield: $78 \%$; mp $196-198^{\circ} \mathrm{C} ; R_{f} 0.82$ (ethyl acetate:ammonia, 9:0.01); IR $\left(\mathrm{KBr}, \mathrm{cm}^{-1}\right): 3320(\mathrm{NH}), 1530$ $(\mathrm{C}=\mathrm{N}), 1070(\mathrm{C}-\mathrm{N}), 2900(\mathrm{C}-\mathrm{H}), 1515(\mathrm{~N}=\mathrm{O}), 3450(\mathrm{O}-\mathrm{H}) ;{ }^{1} \mathrm{H}$ NMR (DMSO, ppm): $\delta 4.62\left(\mathrm{~s}, 2 \mathrm{H}, \mathrm{CH}_{2}\right), 8.62(\mathrm{~s}$, $2 \mathrm{H}$, Schiff bases), 6.92-7.8 (m, 10H, Ar-H), $3.80\left(\mathrm{~s}, 6 \mathrm{H}, \mathrm{OCH}_{3}\right), 2.34\left(\mathrm{~s}, 6 \mathrm{H}, \mathrm{CH}_{3}\right), 9.52(\mathrm{~s}, 2 \mathrm{H}, \mathrm{OH})$; EI-MS: $m / z$ $[\mathrm{M}+\mathrm{H}]^{+}$584; Anal. Calcd for $\mathrm{C}_{31} \mathrm{H}_{28} \mathrm{~N}_{4} \mathrm{O}_{8}: \mathrm{C}, 63.69 ; \mathrm{H}, 4.83 ; \mathrm{N}, 9.58$. Found: C, 63.62; H, 4.72; N, 9.72.

\subsubsection{General procedure for the synthesis of 3,3'-(4,4'-methylene bis(2-methyl-5-nitro-4,1-phenylene)) bis(2- substituted phenylthiazolidin-4-one)}

A mixture of two mole of thioglycolic acid and one mole of 4-4'-methylene bis( $\mathrm{N}$-substituted benzylidene-2methyl-5-nitro aniline) in 1,4-dioxane was refluxed for 12-14 h. The progress of reaction was monitored by $\mathrm{TlC}$ at appropriate time interval. The excess of solvent was distilled off and the solid that separated was collected by filtration and neutralized with sodium bicarbonate to get desired product. The product was recrystallized from ethanol.

3.1.2.1. 3,3'-(4,4'-methylene bis(2-methyl-5-nitro-4,1-phenylene)) bis(2-chloro phenylthiazolidin-4-one) (Sb $\left.{ }_{1}\right)$. Light yellow crystals; yield: $72 \%$; $\mathrm{mp} 272-275^{\circ} \mathrm{C} ; R_{f} 0.69$ (ethyl acetate:ammonia, 9:0.01); IR $\left(\mathrm{KBr}, \mathrm{cm}^{-1}\right): 1177$ (CN), $2990(\mathrm{C}-\mathrm{H}), 1550(\mathrm{~N}=\mathrm{O}), 680(\mathrm{C}-\mathrm{Cl}), 1730(\mathrm{C}=\mathrm{O}), 668(\mathrm{C}-\mathrm{S}) ;{ }^{1} \mathrm{H}$ NMR (DMSO, ppm): $\delta 4.20\left(\mathrm{~s}, 2 \mathrm{H}, \mathrm{CH}_{2}\right)$, 3.60-6.42 (m, $6 \mathrm{H}$, thiazolidinone), 7.10-7.92 (m, $12 \mathrm{H}, \mathrm{Ar}-\mathrm{H}), 2.28\left(\mathrm{~s}, 6 \mathrm{H}, \mathrm{CH}_{3}\right)$; EI-MS: $\mathrm{m} / z[\mathrm{M}+\mathrm{H}]^{+}$709; Anal. Calcd for $\mathrm{C}_{33} \mathrm{H}_{26} \mathrm{Cl}_{2} \mathrm{~N}_{4} \mathrm{O}_{6} \mathrm{~S}_{2}$ : C, 55.86; H, 3.69; N, 7.90. Found: C, 55.70; H, 3.88; N, 7.82.

3.1.2.2. 3,3'-(4,4'-methylene bis(2-methyl-5-nitro-4,1-phenylene)) bis(4-chloro phenylthiazolidin-4-one) (Sb $\left.{ }_{2}\right)$. Light yellow crystals; yield: $72 \%$; mp $262-265^{\circ} \mathrm{C} ; R_{f} 0.74$ (ethyl acetate:ammonia, 9:0.01); IR $\left(\mathrm{KBr}, \mathrm{cm}^{-1}\right): 1188(\mathrm{C}$ $\mathrm{N}), 3010(\mathrm{C}-\mathrm{H}), 1520(\mathrm{~N}=\mathrm{O}), 658(\mathrm{C}-\mathrm{Cl}), 1690(\mathrm{C}=\mathrm{O}), 654(\mathrm{C}-\mathrm{S}) ;{ }^{1} \mathrm{H}$ NMR (DMSO, ppm): $\delta 4.20\left(\mathrm{~s}, 2 \mathrm{H}, \mathrm{CH}_{2}\right)$, 3.60-6.42 (m, 6H, thiazolidinone), 6.90-7.82 (m, 12H, Ar-H), $2.28\left(\mathrm{~s}, 6 \mathrm{H}, \mathrm{CH}_{3}\right)$; EI-MS: $\mathrm{m} / \mathrm{z}[\mathrm{M}+\mathrm{H}]^{+} 709$; Anal. Calcd for $\mathrm{C}_{33} \mathrm{H}_{26} \mathrm{Cl}_{2} \mathrm{~N}_{4} \mathrm{O}_{6} \mathrm{~S}_{2}:$ C, 55.86; H, 3.69; N, 7.90. Found: C, 55.70; H, 3.88; N, 7.82. 
3.1.2.3. 3,3'-(4,4'-methylene bis(2-methyl-5-nitro-4,1-phenylene))bis(4-methyl phenylthiazolidin-4-one) (Sb $\left.{ }_{3}\right)$. Light yellow crystals; yield: $68 \%$; $\mathrm{mp} 282-285^{\circ} \mathrm{C} ; R_{f} 0.68$ (ethyl acetate:ammonia, 9:0.01); IR $\left(\mathrm{KBr}, \mathrm{cm}^{-1}\right)$ : $1075(\mathrm{C}-$ $\mathrm{N}), 3030(\mathrm{C}-\mathrm{H}), 1467(\mathrm{~N}=\mathrm{O}), 1696(\mathrm{C}=\mathrm{O}) ;{ }^{1} \mathrm{H}$ NMR (DMSO, ppm): $\delta 4.20\left(\mathrm{~s}, 2 \mathrm{H}, \mathrm{CH}_{2}\right), 3.60-6.42(\mathrm{~m}, 6 \mathrm{H}$, thiazolidinone), 7.18-8.22 (m, 12H, Ar-H), 2.28-2.39 (m, 12H, $\left.\mathrm{CH}_{3}\right)$; EI-MS: $\mathrm{m} / \mathrm{z}[\mathrm{M}+\mathrm{H}]^{+}$668; Anal. Calcd for $\mathrm{C}_{35} \mathrm{H}_{32} \mathrm{~N}_{4} \mathrm{O}_{6} \mathrm{~S}_{2}:$ C, 62.86; H, 4.82; N, 8.38. Found: C, 62.67; H, 4.89; N, 8.27

3.1.2.4. 3,3'-(4,4'-methylene bis(2-methyl-5-nitro-4,1-phenylene)) bis(2-fluoro phenylthiazolidin-4-one) (Sb $\left.{ }_{4}\right)$. Yellow crystals; yield: $84 \%$; mp $292-293^{\circ} \mathrm{C} ; R_{f} 0.78$ (ethyl acetate:ammonia, 9:0.01); IR $\left(\mathrm{KBr}, \mathrm{cm}^{-1}\right): 1090(\mathrm{C}-\mathrm{N})$, $3010(\mathrm{C}-\mathrm{H}), 1450(\mathrm{~N}=\mathrm{O}), 710(\mathrm{C}-\mathrm{F}), 1710(\mathrm{C}=\mathrm{O}) ;{ }^{1} \mathrm{H}$ NMR (DMSO, ppm): $\delta 4.20\left(\mathrm{~s}, 2 \mathrm{H}, \mathrm{CH}_{2}\right), 3.60-6.42(\mathrm{~m}, 6 \mathrm{H}$, thiazolidinone), 6.92-7.98 (m, 12H, Ar-H), $2.28\left(\mathrm{~s}, 6 \mathrm{H}, \mathrm{CH}_{3}\right)$; EI-MS: $\mathrm{m} / \mathrm{z}[\mathrm{M}+\mathrm{H}]^{+}$677; Anal. Calcd for $\mathrm{C}_{33} \mathrm{H}_{26} \mathrm{~F}_{2} \mathrm{~N}_{4} \mathrm{O}_{6} \mathrm{~S}_{2}$ : C, 58.57; H, 3.87; N, 8.28. Found: C, 58.72; H, 4.03; N, 8.39.

3.1.2.5. 3,3'-(4,4'-methylene bis(2-methyl-5-nitro-4,1-phenylene)) bis(4-fluoro phenylthiazolidin-4-one) (Sb $\left.{ }_{5}\right)$. Yellow crystals; yield: $84 \%$; mp $286-288^{\circ} \mathrm{C} ; R_{f} 0.66$ (ethyl acetate:ammonia, 9:0.01); IR $\left(\mathrm{KBr}, \mathrm{cm}^{-1}\right): 1080(\mathrm{C}-\mathrm{N})$, $3035(\mathrm{C}-\mathrm{H}), 1465(\mathrm{~N}=\mathrm{O}), 730(\mathrm{C}-\mathrm{F}), 1725(\mathrm{C}=\mathrm{O}) ;{ }^{1} \mathrm{H}$ NMR (DMSO, ppm): $\delta 4.20\left(\mathrm{~s}, 2 \mathrm{H}, \mathrm{CH}_{2}\right), 3.60-6.42(\mathrm{~m}, 6 \mathrm{H}$, thiazolidinone), 7.04-8.18 (m, 12H, Ar-H), $2.28\left(\mathrm{~s}, 6 \mathrm{H}, \mathrm{CH}_{3}\right)$; EI-MS: $\mathrm{m} / \mathrm{z}[\mathrm{M}+\mathrm{H}]^{+}$677; Anal. Calcd for $\mathrm{C}_{33} \mathrm{H}_{26} \mathrm{~F}_{2} \mathrm{~N}_{4} \mathrm{O}_{6} \mathrm{~S}_{2}$ : C, 58.57; H, 3.87; N, 8.28. Found: C, 58.72; H, 4.03; N, 8.39.

3.1.2.6. 3,3'-(4,4'-methylene bis(2-methyl-5-nitro-4,1-phenylene)) bis(2-nitro phenylthiazolidin-4-one) (Sb $\left.{ }_{6}\right)$. Yellow crystals; yield: $83 \%$; mp $289-290^{\circ} \mathrm{C} ; R_{f} 0.72$ (ethyl acetate:ammonia, 9:0.01); IR $\left(\mathrm{KBr}, \mathrm{cm}^{-1}\right)$ : $1090(\mathrm{C}-\mathrm{N})$, $3020(\mathrm{C}-\mathrm{H}), 1510(\mathrm{~N}=\mathrm{O}), 1690(\mathrm{C}=\mathrm{O}) ;{ }^{1} \mathrm{H}$ NMR (DMSO, ppm): $\delta 4.20\left(\mathrm{~s}, 2 \mathrm{H}, \mathrm{CH}_{2}\right), 3.60-6.42(\mathrm{~m}, 6 \mathrm{H}$, thiazolidinone), 7.54-8.28 (m, $12 \mathrm{H}, \mathrm{Ar}-\mathrm{H}), 2.28\left(\mathrm{~s}, 6 \mathrm{H}, \mathrm{CH}_{3}\right)$; EI-MS: $\mathrm{m} / \mathrm{z}[\mathrm{M}+\mathrm{H}]^{+} 730$; Anal. Calcd for $\mathrm{C}_{33} \mathrm{H}_{26} \mathrm{~N}_{6} \mathrm{O}_{10} \mathrm{~S}_{2}$ : C, 54.24; H, 3.59; N, 11.50. Found: C, 59.32; H, 3.76; N, 11.38.

3.1.2.7. 3,3'-(4,4'-methylene bis(2-methyl-5-nitro-4,1-phenylene)) bis(4-nitro phenylthiazolidin-4-one) (Sb $\left.{ }_{7}\right)$. Yellow crystals; yield: $73 \%$; mp 296-298 ${ }^{\circ} \mathrm{C} ; R_{f} 0.62$ (ethyl acetate:ammonia, 9:0.01); IR $\left(\mathrm{KBr}, \mathrm{cm}^{-1}\right)$ : $1080(\mathrm{C}-\mathrm{N})$, $3010(\mathrm{C}-\mathrm{H}), 1520(\mathrm{~N}=\mathrm{O}), 1695(\mathrm{C}=\mathrm{O}) ;{ }^{1} \mathrm{H}$ NMR (DMSO, ppm): $\delta 4.20\left(\mathrm{~s}, 2 \mathrm{H}, \mathrm{CH}_{2}\right), 3.60-6.42(\mathrm{~m}, 6 \mathrm{H}$, thiazolidinone), 7.62-8.34 (m, 12H, Ar-H), $2.28\left(\mathrm{~s}, 6 \mathrm{H}, \mathrm{CH}_{3}\right)$; EI-MS: $\mathrm{m} / \mathrm{z}[\mathrm{M}+\mathrm{H}]^{+}$730; Anal. Calcd for $\mathrm{C}_{33} \mathrm{H}_{26} \mathrm{~N}_{6} \mathrm{O}_{10} \mathrm{~S}_{2}$ : C, 54.24; H, 3.59; N, 11.50. Found: C, 59.32; H, 3.76; N, 11.38.

3.1.2.8.3,3'-(4,4'-methylenebis(2-methyl-5-nitro-4,1-phenylene))bis(2-hydroxy phenylthiazolidin-4-one) (Sb ( $_{8}$. Yellow crystals; yield: $67 \%$; $\mathrm{mp} 276-278^{\circ} \mathrm{C} ; R_{f} 0.71$ (ethyl acetate:ammonia, 9:0.01); IR $\left(\mathrm{KBr}, \mathrm{cm}^{-1}\right): 3430(\mathrm{O}-\mathrm{H})$, $1065(\mathrm{C}-\mathrm{N}), 2995(\mathrm{C}-\mathrm{H}), 1515(\mathrm{~N}=\mathrm{O}), 1735$ (C=O); ${ }^{1} \mathrm{H}$ NMR (DMSO, ppm): $\delta 4.20$ (s, 2H, $\left.\mathrm{CH}_{2}\right), 3.60-6.42(\mathrm{~m}, 6 \mathrm{H}$, thiazolidinone), 6.62-7.94 (m, 12H, Ar-H), $2.28\left(\mathrm{~s}, 6 \mathrm{H}, \mathrm{CH}_{3}\right), 9.18(\mathrm{~s}, 2 \mathrm{H}, \mathrm{OH})$; EI-MS: $\mathrm{m} / \mathrm{z}[\mathrm{M}+\mathrm{H}]^{+}$672; Anal. Calcd for $\mathrm{C}_{33} \mathrm{H}_{28} \mathrm{~N}_{4} \mathrm{O}_{8} \mathrm{~S}_{2}$ : C, 58.92; H, 4.20; N, 8.33. Found: C, 58.76; H, 4.28; N, 8.29.

3.1.2.9. 3,3'-(4,4'-methylene bis(2-methyl-5-nitro-4,1-phenylene)) bis(2-hydroxy-3-methoxy phenylthiazolidin-4one) $\left(\boldsymbol{S b}_{9}\right)$. Yellow crystals; yield: $77 \% ; \mathrm{mp} 276-278^{\circ} \mathrm{C} ; R_{f} 0.68$ (ethyl acetate:ammonia, 9:0.01); IR $\left(\mathrm{KBr}, \mathrm{cm}^{-1}\right)$ : $3450(\mathrm{O}-\mathrm{H}), 1055(\mathrm{C}-\mathrm{N}), 2970(\mathrm{C}-\mathrm{H}), 1530(\mathrm{~N}=\mathrm{O}), 1740(\mathrm{C}=\mathrm{O})$; ${ }^{1} \mathrm{H}$ NMR (DMSO, ppm): $\delta 4.20\left(\mathrm{~s}, 2 \mathrm{H}, \mathrm{CH}_{2}\right), 3.60-$ $6.42(\mathrm{~m}, 6 \mathrm{H}$, thiazolidinone), 6.76-7.99 (m, 12H, $\mathrm{Ar}-\mathrm{H}), 2.28\left(\mathrm{~s}, 6 \mathrm{H}, \mathrm{CH}_{3}\right), 9.78(\mathrm{~s}, 2 \mathrm{H}, \mathrm{OH}), 3.92\left(\mathrm{~m}, 6 \mathrm{H}, \mathrm{OCH}_{3}\right)$; EI-MS: $\mathrm{m} / z[\mathrm{M}+\mathrm{H}]^{+} 732$; Anal. Calcd for $\mathrm{C}_{35} \mathrm{H}_{32} \mathrm{~N}_{4} \mathrm{O}_{10} \mathrm{~S}_{2}$ : C, 58.37; $\mathrm{H}, 4.40 ; \mathrm{N}, 7.65$. Found: $\mathrm{C}, 58.56 ; \mathrm{H}, 4.28 ; \mathrm{N}$, 7.59 .

3.1.2.10. 3,3'-(4,4'-methylene bis(2-methyl-5-nitro-4,1-phenylene)) bis(4-hydroxy-3-methoxy phenylthiazolidin4-one) $\left(\boldsymbol{S} \boldsymbol{b}_{10}\right)$. Yellow crystals; yield: $69 \% ; \mathrm{mp} 266-268^{\circ} \mathrm{C} ; R_{f} 0.60$ (ethyl acetate:ammonia, 9:0.01); $\mathrm{IR}\left(\mathrm{KBr}, \mathrm{cm}^{-1}\right)$ : $3455(\mathrm{O}-\mathrm{H}), 1085(\mathrm{C}-\mathrm{N}), 2980(\mathrm{C}-\mathrm{H}), 1525(\mathrm{~N}=\mathrm{O}), 1735(\mathrm{C}=\mathrm{O})$; ${ }^{1} \mathrm{H}$ NMR (DMSO, ppm): $\delta 4.20\left(\mathrm{~s}, 2 \mathrm{H}, \mathrm{CH}_{2}\right), 3.60-$ $6.42\left(\mathrm{~m}, 6 \mathrm{H}\right.$, thiazolidinone), 6.82-8.09 (m, 12H, Ar-H), $2.28\left(\mathrm{~s}, 6 \mathrm{H}, \mathrm{CH}_{3}\right), 9.78(\mathrm{~s}, 2 \mathrm{H}, \mathrm{OH}), 3.72\left(\mathrm{~m}, 6 \mathrm{H}, \mathrm{OCH}_{3}\right)$; EI-MS: $\mathrm{m} / z[\mathrm{M}+\mathrm{H}]^{+} 732$; Anal. Calcd for $\mathrm{C}_{35} \mathrm{H}_{32} \mathrm{~N}_{4} \mathrm{O}_{10} \mathrm{~S}_{2}$ : C, 58.37; H, 4.40; N, 7.65. Found: $\mathrm{C}, 58.56 ; \mathrm{H}, 4.28 ; \mathrm{N}$, 7.59 . 
<smiles>Cc1ccc([N+](=O)[O-])cc1N</smiles>

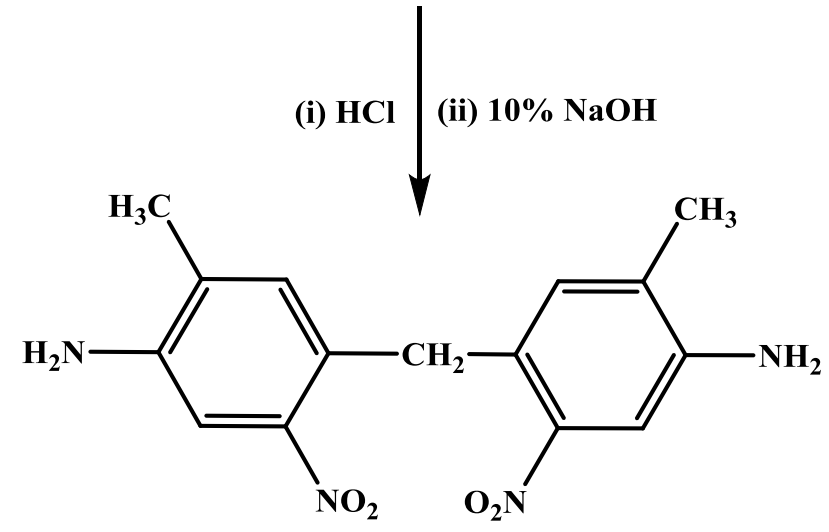

4,4'-Methylene bis(2-methyl-5-nitro aniline)

Scheme 1
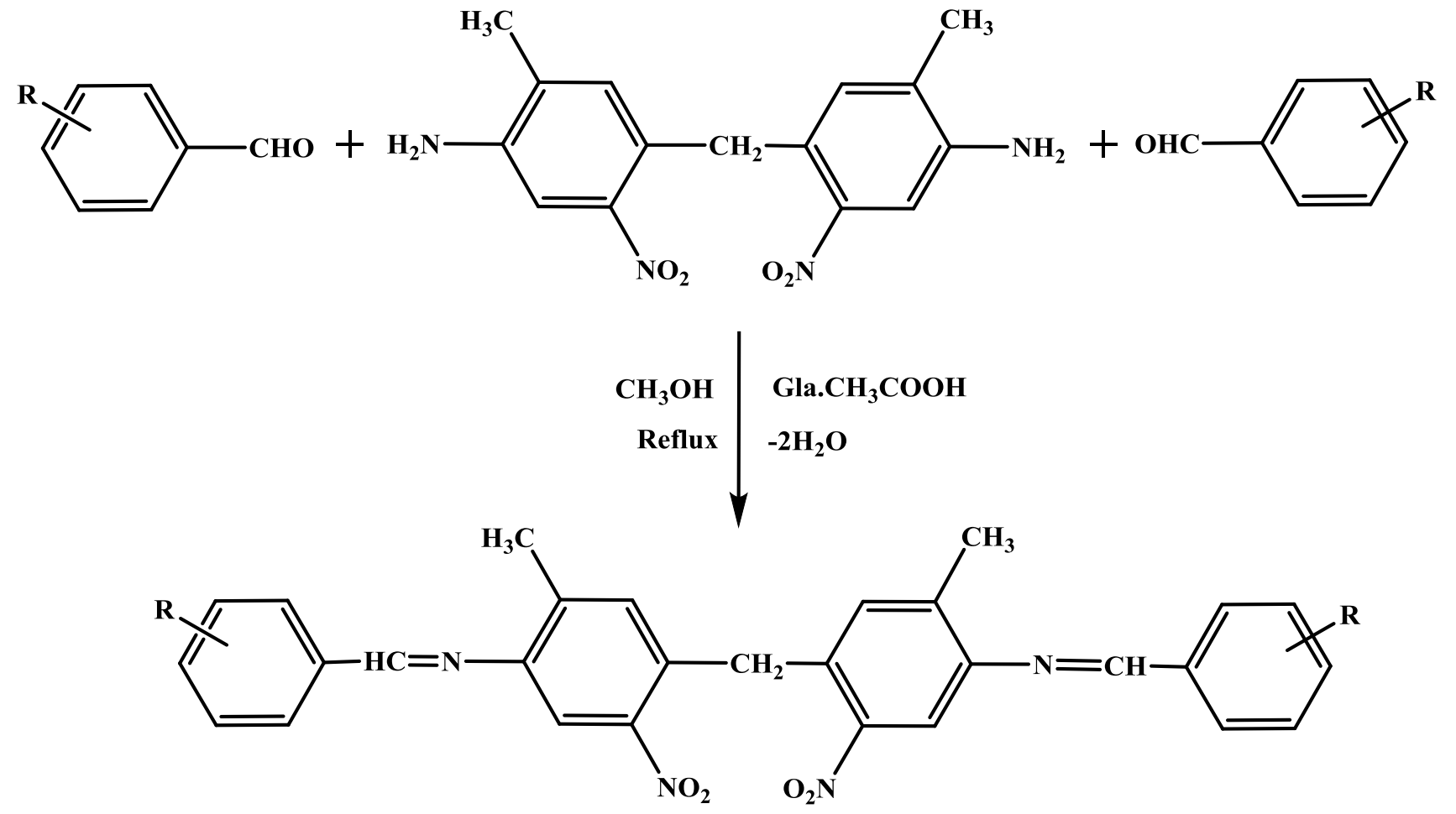

Where, $\mathrm{R}=2-\mathrm{Cl}, 4-\mathrm{Cl}, 4-\mathrm{CH}_{3}, 2-\mathrm{F}, 4-\mathrm{F}, 2-\mathrm{NO}_{2}, 4-\mathrm{NO}_{2}, 2-\mathrm{OH}, 2-\mathrm{OH}-3-\mathrm{OCH}_{3}, 4-\mathrm{OH}-3-\mathrm{OCH}_{3}$

$\mathrm{Sa}_{1}$ to $\mathrm{Sa}_{10}$

Scheme 2:- 

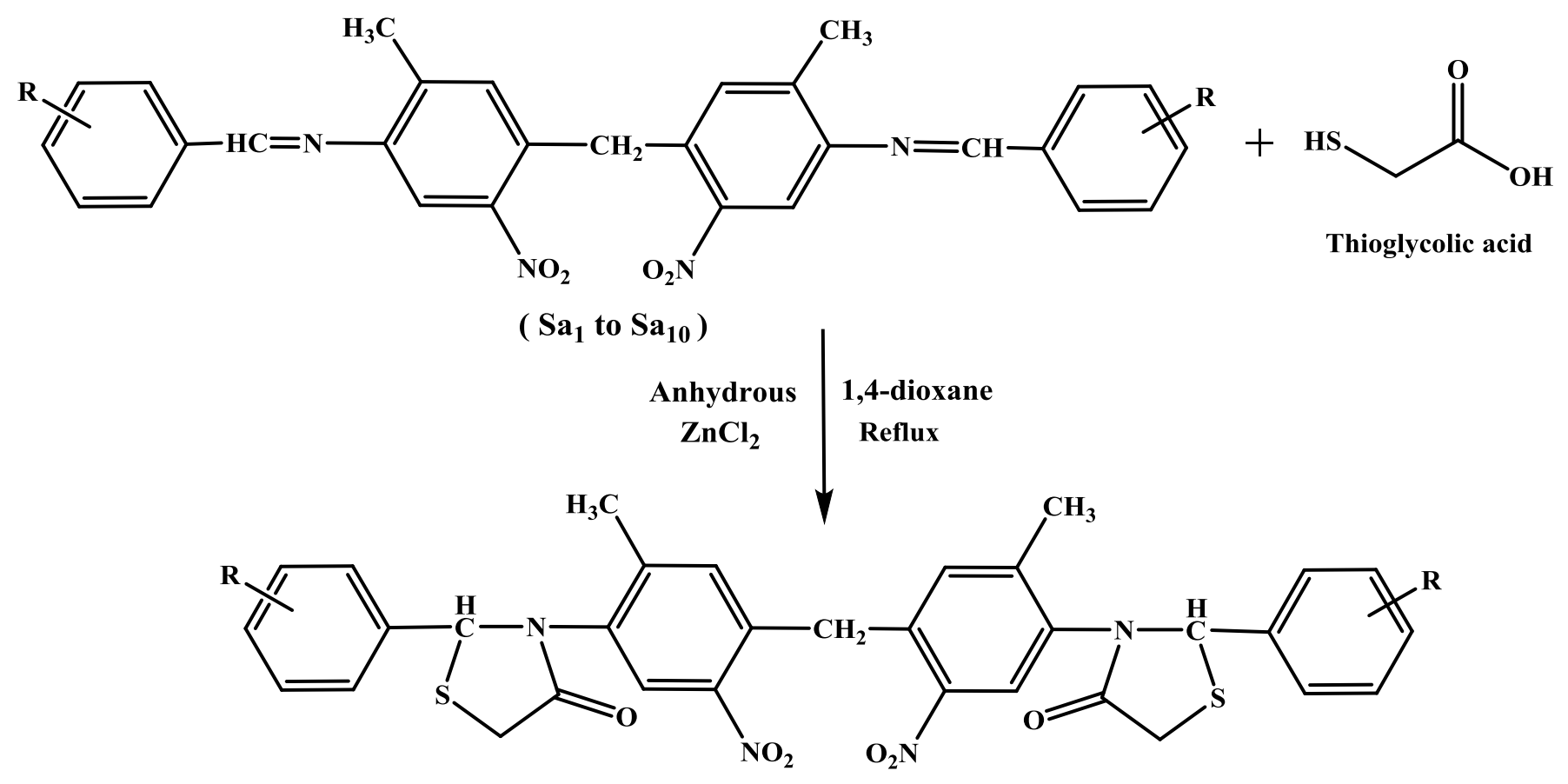

Where, $\mathrm{R}=2-\mathrm{Cl}, 4-\mathrm{Cl}, 4-\mathrm{CH}_{3}, 2-\mathrm{F}, 4-\mathrm{F}, 2-\mathrm{NO}_{2}, 4-\mathrm{NO}_{2}, 2-\mathrm{OH}, 2-\mathrm{OH}-3-\mathrm{OCH}_{3}, 4-\mathrm{OH}-3-\mathrm{OCH}_{3}$

$\left(\mathrm{Sb}_{1}\right.$ to $\left.\mathrm{Sb}_{10}\right)$

\section{Results and Discussion:-}

Scheme 3:-

\section{Antibacterial Activity:-}

The stock solution $(1000 \mathrm{lg} / \mathrm{mL})$ of the test compounds was prepared by dissolving test compounds $(10 \mathrm{mg})$ in dimethylsulfoxide (DMSO) $(10 \mathrm{~mL}$ ). The stock solution was sterilized by passing through a $0.2 \mathrm{~mm}$ polycarbonate sterile membrane (Nucleopore) filter. Further, serial dilutions of the test compounds were carried out and the following concentrations were used 1000, 500, 250,125, 62, 32, 16, 8, 4 and $1 \mathrm{lg} / \mathrm{mL}$. Test compounds at various concentrations were added to culture medium in a sterilized borosilicate test tube and different bacterial strains were inoculated at 106 bacilli $/ \mathrm{mL}$ concentration. A control was also prepared for the plates in the same way using solvent DMSO. The tubes were incubated at room temperature for $24 \mathrm{~h}$ and then examined for the presence or absence of growth of the test organisms. All experiments were performed in triplicate. The MIC values were obtained from the lowest concentration of the test compounds where the tubes remained clear (i.e. no turbidity), indicating that the bacterial growth was completely inhibited at this concentration. The MIC values were also determined for the wellknown antibiotics (ciprofloxacin and ampicillin) to compare the antibacterial activity of our test compounds with the antibiotics, which are currently in therapy. The MIC values are expressed in $\mathrm{gg} / \mathrm{mL}$ and summarized in Table 1 .

\section{Antifungal Activity:-}

Sabouraud Dextrose Agar (Merck) media were used for the cultivation of fungi. Normal saline was used to make a suspension of spore of fungal strain. A loopful of particular fungal strain was transferred to $3 \mathrm{~mL}$ saline to get a suspension of corresponding species. A solution of agar media $(20 \mathrm{~mL})$ was poured into each petri dish. Excess of suspension was decanted and the plates were dried. After drying, wells were made using an agar punch and test samples, reference standard and negative control (DMSO) were placed in labeled wells in each petri plate. The petri plates were incubated at room temperature for $48 \mathrm{~h}$. The MIC values were noted and the activity of each compound was compared with fluconazole as standard drug. The results of antifungal activity are given in MIC values as $\lg / \mathrm{mL}$ and are illustrated in Table 1. 
Table 1:- Antibacterial and antifungal activities of compounds $S a_{1}-S b_{10}$.

\begin{tabular}{|c|c|c|c|c|c|c|}
\hline \multirow[t]{2}{*}{ compound } & \multicolumn{2}{|c|}{ Gram negative bacteria } & \multicolumn{2}{|c|}{ Gram positive bacteria } & \multicolumn{2}{|l|}{ Fungi } \\
\hline & E. coli & P. aeruginosa & S. aureus & subtilis & C. albicans & A. niger \\
\hline$S a_{1}$ & 54 & 47 & 77 & 59 & 65 & 52 \\
\hline $\mathrm{Sa}_{2}$ & 58 & 40 & 57 & 86 & 74 & 88 \\
\hline $\mathrm{Sa}_{3}$ & $>100$ & $>100$ & 86 & 72 & 92 & $>100$ \\
\hline $\mathrm{Sa}_{4}$ & 05 & 03 & 36 & 28 & 06 & 09 \\
\hline $\mathrm{Sa}_{5}$ & 08 & 10 & 24 & 38 & 05 & 03 \\
\hline$S a_{6}$ & 09 & 06 & 52 & 44 & 46 & 55 \\
\hline $\mathrm{Sa}_{7}$ & 03 & 04 & 39 & 33 & 29 & 37 \\
\hline$S a_{8}$ & 69 & 52 & 18 & 24 & 21 & 17 \\
\hline$S a_{9}$ & 64 & 55 & 04 & 06 & 42 & 32 \\
\hline$S a_{10}$ & 07 & 12 & 09 & 12 & 28 & 39 \\
\hline$S b_{1}$ & 28 & 39 & 68 & 64 & 68 & 88 \\
\hline$S b_{2}$ & 37 & 42 & 76 & 87 & 77 & 97 \\
\hline$S b_{3}$ & 82 & 66 & 82 & 90 & $>100$ & 82 \\
\hline $\mathrm{Sb}_{4}$ & 17 & 14 & 29 & 22 & 12 & 08 \\
\hline$S b_{5}$ & 11 & 28 & 14 & 19 & 04 & 03 \\
\hline$S b_{6}$ & 07 & 05 & 32 & 38 & 52 & 44 \\
\hline $\boldsymbol{S} \boldsymbol{b}_{7}$ & 03 & 03 & 48 & 52 & 28 & 38 \\
\hline$S b_{8}$ & 37 & 45 & 19 & 15 & 35 & 46 \\
\hline$S b_{9}$ & 27 & 15 & 05 & 04 & 29 & 19 \\
\hline$S b_{10}$ & 38 & 25 & 08 & 10 & 20 & 28 \\
\hline Ciprofloxacin & 03 & 04 & 05 & 02 & - & - \\
\hline Ampicillin & 02 & 02 & 01 & 01 & - & - \\
\hline Fluconazole & - & - & - & - & 01 & 02 \\
\hline
\end{tabular}

All activity data are given as MIC $(\mu \mathrm{g} / \mathrm{mL})$. E. coli: Escherichia coli; P. aeruginosa: Pseudomonas aeruginosa; S. aureus: Staphylococcus aureus; B. subtilis: Bacillus subtilis; C. albicans: Candida albicans; A. niger: Aspergillus niger.

\section{Conclusion:-}

A series of newly Schiff bases and thiazolidinone derivatives were synthesized and characterized by elemental analysis, IR, NMR, and mass spectrometry studies. All the newly synthesized compounds were screened for their antibacterial and antifungal activities by the method of minimum inhibitory concentration (MIC). Antimicrobial study reveals that compound $\mathrm{Sa}_{7}$ and $\mathrm{Sb}_{7}$ having p-nitro substitution exhibited maximum inhibition against microorganism E. coli and $P$. aeruginosa. Similarly, compounds $\mathrm{Sa}_{9}$ and $\mathrm{Sb}_{9}$ also showed significant activity against microorganism $S$. aureus and B. subtilis, which have o-methoxy substituent on the phenyl ring, respectively. The test results showed that compounds $\mathrm{Sa}_{4}, \mathrm{Sa}_{5}$ and $\mathrm{Sb}_{5}$ exhibited significant antifungal activity while remaining compounds showed moderate activity.

\section{Acknowledgement:-}

The authors are grateful to The Head, Department of Chemistry, Faculty of Science, Veer Narmad South Gujarat University, Surat, Gujarat, India for laboratory facilities. We gratefully acknowledge for financial assistance given by University Grants Commission (UGC).

\section{References:-}

1. A. Juan, P. Yaricruz, B. Alina, C. Juan, J Med Chem 6 (2016) 467-473.

2. T. S. Eman, Int J Current Res Chem Pharm Sci 3 (2016) 118-123.

3. A. K. Ahmad Sabry, F. Rabie Saad, M. A. Alaa El-Dine, A. J. A. C. 7 (2016) 233-245.

4. M.R. Ummer, M. Dharmasivam, K. H. Azees, P.N. Rakesh, D. Mukesh, K.R Aziz, New J. Chem.40 (2016) 2451-2465.

5. M. Nath, P.K. Saini, Dalton. Trans. 40 (2011) 7077-7121.

6. E. I. Grace, I. Terungwa, S.O Olajire, S. Dooshima, K. Mabel Iyuana, I.J.I.S.R.18 (2015) 117-121.

7. M.A. Mokhles, A.L. Ammar , A.M.Hanan A.M. Samia, M.A. Mamdouh, A.E.Ahmed BENISEUFUNIV.J.APPL.SCI. 5 (2 0 o 1 6 6) 85-96. [8] K.V. Shuvaev, L.N. Dawe, L.K. Thompson, Eur. J. Inorg. Chem. 29(2010) 4583-4586. 
8. A. Pandey, D.S. Verma, A. Mishra, R.D. Dubey,Int. J. Chem. Tech. Res. 3(2011)178-184.

9. C. Chandramouli, M. R. Shivanand, T. B. Nayanbhai, B. Bheemachari, R. H. Udupi, J. Chem. Pharm. Res .4 (2012)151- 1159.

10. R. P. Chinnasamy, R. Sundararajan, S. Govindaraj,J. Adv. Pharm. Technol. Res.1(2010) 342-347.

11. Barreca, M. L.; Chimirri, A.; De Luca, L.; Monforte, A. M.; Monforte, P.; Rao, A.; Zappala, M.; Balzarini, J.; De Clercq, E.; Pannecouque, C.; Witvrouw, M. Bioorg. Med. Chem. lett. 2001, 11 (13), 1793-6.

12. Amin, K. M.; Rahman, D. E.; Al-Eryani, Y. A. Bioorg. Med. Chem. 2008, 16 (10), 537788.

13. Bhandari, S. V.; Bothara, K. G.; Patil, A. A.; Chitre, T. S.; Sarkate, A. P.; Gore, S. T.; Dangre, S. C.; Khachane, C. V. Bioorg. Med. Chem. 2009, 17 (1), 390-400.

14. Ottana, R.; Mazzon, E.; Dugo, L.; Monforte, F.; Maccari, R.; Sautebin, L.; De Luca, G.; Vigorita, M. G.; Alcaro, S.; Ortuso, F.; Caputi, A. P.; Cuzzocrea, S. Eur. J. Pharmacology. 2002, 448 (1), 71-80.

15. Ottana, R.; Maccari, R.; Giglio, M.; Del Corso, A.; Cappiello, M.; Mura, U.; Cosconati, S.; Marinelli, L.; Novellino, E.; Sartini, S.; La Motta, C.; Da Settimo, F. Eur. J. Med. Chem. 2011, 46 (7), 2797-806.

16. Joy, M. J.; Jacob, N.; Kutty, N. G. Indian Drugs. 2005, (42), 47.

17. Felise, H. B.; Nguyen, H. V.; Pfuetzner, R. A.; Barry, K. C.; Jackson, S. R.; Blanc, M. P.; Bronstein, P. A.; Kline, T.; Miller, S. I. Cell host \& microbe. 2008, 4 (4), 325-36.

18. Aridoss, G.; Amirthaganesan, S.; Kim, M. S.; Kim, J. T.; Jeong, Y. T., Eur. J. Med. Chem. 2009, 44 (10), 4199-210.

19. Carlson, E. E.; May, J. F.; Kiessling, L. L. Chemistry \& biology 2006, 13 (8), 825-37.

20. Wrobel, J.; Jetter, J.; Kao, W.; Rogers, J.; Di, L.; Chi, J.; Perez, M. C.; Chen, G. C.; Shen, E. S. Bioorg. Med. Chem. 2006, 14 (16), 5729-41.

21. Panico, A. M.; Vicini, P.; Geronikaki, A.; Incerti, M.; Cardile, V.; Crasci, L.; Messina, $\quad$ R.; Ronsisvalle, S. Bioorg. Chemistry. 2011, 39 (1), 48-52.

22. Mazzoni, O.; di Bosco, A. M.; Grieco, P.; Novellino, E.; Bertamino, A.; Borrelli, F.; Capasso, R.; Diurno, M. V. Chem. Biol. Drug. Des. 2006, 67 (6), 432-6. 15

23. Jones, R.N., Barry, A.L., Gavan, T.L., Washington, J.A., 1991. In: Lennette, E.H., Balows, A., Hausler, W.J., Shadomy, H.J. (Eds.), A Manual of Clinical Microbiology. American Society of Microbiology, Washington, DC, USA

24. Khan, Z.K., 1997. In: Proceeding International Workshop. UNIDOCDRI, Lucknow, India. 